\title{
GLIDEBENDING OF GENERAL CAPS: AN INFINITESIMAL TREATMENT
}

\author{
EDGAR KANN
}

\begin{abstract}
We prove that two $C^{3}$ isometric surfaces $S^{\prime}$ and $S^{\prime \prime}$ with (not necessarily planar) boundary, immersed in $E^{3}$, with $K>0$, having their spherical images in the same open hemisphere, and which are oriented so that the mean curvatures have the same sign, are congruent if they satisfy the glidebending boundary condition:

Let $\{n: e \cdot n>0, e=$ const. vector $\}$ be the open hemisphere. Then $e \cdot X^{\prime}=$ $e \cdot X^{\prime \prime}$ at corresponding boundary points, where $X^{\prime}$ and $X^{\prime \prime}$ are position vectors of $S^{\prime}$ and $S^{\prime \prime}$.

The method uses the fact that the surface $\frac{1}{2}\left(X^{\prime \prime}-X^{\prime}\right)$ is an infinitesimal bending field for the mean surface $\frac{1}{2}\left(X^{\prime \prime}+X^{\prime}\right)$ and is elementary in that it uses the rotation vector of classical infinitesimal bending theory but no integral formulas, maximum principles for elliptic operators or index theorems. The surfaces considered need not have a simple projection on a plane, be convex in the large (an example is given) nor be simply connected. We use the method to prove the finite and infinitesimal rigidity of general caps and caps under glidebending.
\end{abstract}

1. Introduction. The difference surface of two intrinsically isometric surfaces gives a bending field for their mean surface. That is, if $X^{\prime}$ and $X^{\prime \prime}$ are position vectors of two isometric surfaces and $X=\frac{1}{2}\left(X^{\prime}+X^{\prime \prime}\right), Z=\frac{1}{2}\left(X^{\prime \prime}-X^{\prime}\right)$ then $d X \cdot d Z=0$, which is the condition that $Z$ be an infinitesimal bending field on the mean surface $X$. This fact has been used by Cohn-Vossen [1] in his well-known proof of the congruence of intrinsically isometric ovaloids.

If $X$ were regular in the large then classical infinitesimal methods could be used to prove finite rigidity theorems. However, regularity clearly does not hold in general. Nevertheless, the mean surface for two isometric surfaces whose spherical normal images lie in a hemisphere is regular on certain sets, namely those on which the difference surface is singular. In [3] the author was able, by taking advantage of this, to prove the congruence of two isometric, positively curved, but not necessarily convex, surfaces satisfying this hemisphere condition whose boundary curves are identical. The original bending field $Z$, which of course depends on the relative positions of the surfaces, is replaced by one which differs from it by a trivial bending, i.e., the velocity field of a rigid motion, in such a way that the singular locus becomes a smooth curve or curves. Along such a curve the differential of the new bending is normal to the surface and always points to the same side. This leads to a contradiction if the singular locus is a simple closed curve.

Received by the editors December 23, 1980 and, in revised form, May 12, 1981.

1980 Mathematics Subject Classification. Primary 53A05; Secondary 53C45.

Key words and phrases. Finite rigidity, infinitesimal rigidity, glidebending, bending field, general cap, cap, isometric surface, congruent surface, rotation field, monotypy. 
The method is elementary in the sense that it uses only the classical tools of infinitesimal bending theory, in particular the rotation vector, and does not use integral formulas, maximum principles for elliptic operators or index theorems.

The present paper applies the method to the much weaker boundary condition in which the vector connecting boundary points which correspond under the isometry is only required to be orthogonal to a fixed direction $e$ (glidebending condition) such that $e \cdot n^{\prime}>0$ and $e \cdot n^{\prime \prime}>0$, where $n^{\prime}$ and $n^{\prime \prime}$ are unit surface normals to the surfaces. Although congruence has been proved (see the standard reference [2, \$79]) in the case where the boundary curves lie in a plane, that is, for caps, the result for nonplanar boundaries, that is, for general caps, is a natural generalization whose proof is, to the author's knowledge, new (see $\$ 3$ for definitions). Certainly, the application of the author's methods to the solution of this problem is new.

The glidebending problem is considered in $[2, \S 80]$ for general caps but not proved there. The surfaces considered here are even more general than the general caps in that they need not have a one-to-one projection on some plane, nor be convex in the large (they are locally convex), nor be simply connected.

Theorem 1, which is a monotypy result in the class of $C^{3}$ surfaces, is closely related to a theorem of Pogorelov [5, Theorem 2, p. 178]. Pogorelov proves the theorem for general convex surfaces which have a simple projection on some plane. In $\$ 3$ we give an example of a surface satisfying the hypotheses of Theorem 1 which has no simple projection on a plane and is locally convex but not convex in the large. The infinitesimal rigidity under glidebending of the class of surfaces considered in Theorem 1 is proved by the same methods in $\$ 4$.

In order to convince the reader that the glidebending condition leads to a significant class of problems we refer him to the example in [2, p. 169] of two convex isometric surfaces with plane boundary which are not congruent under rigid motions or reflections. These surfaces do not satisfy the condition $\mathrm{H} 3$ of Theorem 1.

\section{Main result.}

THEOREM 1. Let $M$ be a compact two-dimensional, orientable Riemannian manifold with boundary such that

H1. $M$ is of differentiability class $C^{3}$ and

H2. $M$ has Gauss curvature $K>0$.

Let $S^{\prime}$ and $S^{\prime \prime}$ be isometric immersions of $M$ of class $C^{3}$ in $E^{3}$ such that $\partial S^{\prime}$ and $\partial S^{\prime \prime}$ are $C^{\prime \prime}$ curves.

Let $S^{\prime}$ be oriented so that its second fundamental form is positive definite. We assume that the orientation induced on $S^{\prime \prime}$ by the isometry makes its second fundamental form also positive definite.

Suppose there exists a constant unit vector e such that:

H3. $e \cdot n^{\prime}>0$ and $e \cdot n^{\prime \prime}>0$ on $M+\partial M$, where $n^{\prime}$ and $n^{\prime \prime}$ are the unit surface normal vectors to $S^{\prime}$ and $S^{\prime \prime}$ and 
H4. $e \cdot X^{\prime}=e \cdot X^{\prime \prime}$ on $\partial M$, where $X^{\prime}=X^{\prime}(M)$ and $X^{\prime \prime}=X^{\prime \prime}(M)$ are position vectors of $S^{\prime}$ and $S^{\prime \prime}$ representing the immersion.

Then $S^{\prime}$ and $S^{\prime \prime}$ are congruent under rigid body motions.

An isometry satisfying the boundary condition $\mathrm{H} 4$ is called a glidebending $[2, \S 80]$.

Proof. Choose an arbitrary point $P$ on $\partial M$ and let $u, v$ be local parameters in a neighborhood of $P$ with $v=0$ representing $\partial M$. Since by definition $Z=$ $\frac{1}{2}\left(X^{\prime \prime}-X^{\prime}\right), \mathrm{H} 4$ is equivalent to $e \cdot Z=0$ on $\partial M$. Hence

$$
e \cdot Z_{u}=0 \text { on } \partial M
$$

Conditions $\mathrm{H} 1-\mathrm{H} 4$ are invariant under translations of $S^{\prime}$ and $S^{\prime \prime}$ perpendicular to $e$ as well as rotations about an axis parallel to $e$. Therefore we may place the surfaces by a rigid motion so that

$$
Z(P)=0 \quad \text { and } \quad Z_{u}(P)=0 .
$$

Calculation using $X=\frac{1}{2}\left(X^{\prime}+X^{\prime \prime}\right)$ shows that, for any two isometric surfaces, $X_{u} \times X_{v}+Z_{u} \times Z_{v}=\frac{1}{2}\left(X_{u}^{\prime} \times X_{v}^{\prime}+X_{u}^{\prime \prime} \times X_{v}^{\prime \prime}\right)$ and hence by H3

$$
e \cdot X_{u} \times X_{v}+e \cdot Z_{u} \times Z_{v}>0 \text { on } M+\partial M \text {. }
$$

Therefore the mean surface $X$ is regular at any point where $Z_{u} \times Z_{v}=0$ and, in particular, at $P$. Hence there is a neighborhood $N_{p}$ of $P$ on which $X$ is regular. As mentioned above $d X \cdot d Z=0$ so $Z$ is a bending field on $X$. A classical result of the theory of infinitesimal bending [2, p. 54] states that there exists a rotation vector $y$ defined at any regular point of $X$ such that

$$
y \times d X=d Z \text {. }
$$

We will next show that $n^{\prime}=n^{\prime \prime}$ at $P$. Since $X_{u}^{\prime}=X_{u}^{\prime \prime}$ at $P$ by (2.2), it will follow that $X_{v}^{\prime}=X_{v}^{\prime \prime}$. Since $P$ is an arbitrary point of $\partial M$ and since $e \cdot n^{\prime}, e \cdot n^{\prime \prime}, e \cdot X_{v}^{\prime}$ and $e \cdot X_{v}^{\prime \prime}$ are invariant under a translation of $X^{\prime}$ or $X^{\prime \prime}$ perpendicular to $e$ or a rotation about an axis parallel to $e$, it will follow that

$$
e \cdot n^{\prime}=e \cdot n^{\prime \prime} \text { and } e \cdot X_{v}^{\prime}=e \cdot X_{v}^{\prime \prime} \text { on } \partial M
$$

From there it is easy to show that the boundary strips of $S^{\prime}$ and $S^{\prime \prime}$ are congruent and hence that $S^{\prime}$ and $S^{\prime \prime}$ are congruent.

To show that $n^{\prime}=n^{\prime \prime}$ we suppose that $n^{\prime} \neq n^{\prime \prime}$ at $P$ and derive a contradiction. By (2.4) and (2.2) there is a vector $y_{P}$ such that $y_{P} \times X_{u}=Z_{u}=0$ and $y_{P} \times X_{v}=$ $Z_{v}$ at $P$. $Z_{v} \neq 0$ since $n^{\prime} \neq n^{\prime \prime}$; hence $y_{P} \neq 0$. Since $y_{P}$ is parallel to $X_{u}, y_{P} \cdot n_{P}=0$, where $n_{P}$ is the unit surface normal to $X$ at $P$.

Next we will show that $P$ is an endpoint of a smooth segment in $M$ on which $y \cdot n=0$.

$$
(y \cdot n)_{u}=y \cdot n_{u} \text { and }(y \cdot n)_{v}=y \cdot n_{v}
$$

follow from the basic formulas of infinitesimal bending theory, $y_{u}=\alpha X_{u}-\beta X_{v}$ and $y_{v}=\gamma X_{u}-\alpha X_{v}$. 
By hypothesis the second fundamental forms of $X^{\prime}$ and $X^{\prime \prime}$ are positive definite. The coefficients of the second fundamental form of the mean surface $X$ are

$$
\begin{gathered}
L=\frac{1}{2}\left(L^{\prime}+L^{\prime \prime}\right) \cos \omega, \quad M=\frac{1}{2}\left(M^{\prime}+M^{\prime \prime}\right) \cos \omega, \\
N=\frac{1}{2}\left(N^{\prime}+N^{\prime \prime}\right) \cos \omega,
\end{gathered}
$$

where the primed quantities are the coefficients of the second fundamental forms of $X^{\prime}$ and $X^{\prime \prime}$ and where $\cos \omega=n^{\prime} \cdot n=n^{\prime \prime} \cdot n$ and $\cos \omega>0$ if $X$ is regular [2,p. 141]. Therefore where $X$ is regular its second fundamental form is positive definite.

Not both terms in (2.6) can vanish at $P$ since $n_{u}, n_{v}$, and $n$ are linearly independent and $y_{P} \neq 0$. Hence it follows from the Implicit Function Theorem that there is a half-open $C^{\prime \prime}$ segment with one endpoint at $P$ on which $y \cdot n=0$. This segment is not tangent to $\partial M$ at $P$ for, since $u$ is a parameter on $\partial M$, $(y \cdot n)_{u}=y \cdot n_{u}=0$. But $y \times X_{u}=Z_{u}=0$ at $P$, so $X_{u}$ is parallel to $y$. This contradicts $X_{u} \cdot n_{u} \neq 0$. Therefore the half-open segment enters $M$ and has its other endpoint in $M$.

By (2.4)

$$
Z_{u} \times Z_{v}=\left(y \cdot X_{u} \times X_{v}\right) y
$$

so $y \cdot n=0$ is equivalent to $Z_{u} \times Z_{v}=0$ at any regular point of $X$. Therefore $Z_{u} \times Z_{v}=0$ at every point of the half-open segment and hence, by continuity, equals zero at the other end also. By (2.3) $X$ is regular at the other endpoint so $y \cdot n$ is defined and equals zero there. If $y$ never vanishes the argument may be repeated, extending the segment to a curve which must end on $\partial M$ since, as can be seen from elementary compactness arguments, it cannot intersect itself or spiral toward a closed curve in $M$.

We next replace the bending field $Z$ by a bending field which differs from $Z$ by a trivial bending field in such a way that the corresponding rotation field never vanishes and so that the preceding argument can be carried out for the new field.

Define $Z_{g}=Z-g \times X$ where $g$ is a constant vector to be chosen appropriately. $d z_{g}=(y-g) \times d X$, so $y-g$ is the rotation vector for $Z_{g}$. The surface described by the position vector $y$ cannot contain an open subset of $E^{3}$. Therefore there exist vectors $g$ of arbitrarily small absolute value such that $y-g$ never vanishes on that part of $M+\partial M$ where $X$ is regular.

Choose local parameters $u, v$ in a neighborhood of $P$ so that $P$ has coordinates $u=0, v=0, \partial M$ has the equation $v=0$, and the half-open segment on which $y \cdot n=0$ has the equation $u=0$. Apply the Implicit Function Theorem to the function $f$ defined by $f(u, v, g)=(y-g) \cdot n \cdot f_{u}(0,0,0)=\left.y \cdot n_{u}\right|_{P}, f_{v}(0,0,0)=$ $\left.y \cdot n_{v}\right|_{P}$ and $f(0,0,0)=\left.y \cdot n\right|_{P}=0$. By the reasoning above we know that $f_{u}(0,0,0)$ $\neq 0$ and therefore there exists a $C^{\prime \prime}$ function $u=h(v, g)$ defined in some neighborhood $N$ of $(0,0)$ such that $f(h(v, g), v, g)=0$ and $h(0,0)=0$. In other words $(y-g) \cdot n=0$ on a segment whose equation, for each fixed $g$ of sufficiently small absolute value, is $u=h(v, g)$. Since $h$ is differentiable, the segment is not tangent to $\partial M$ at any point $(u, 0)$ on $\partial M$. Hence there is a half-open segment with one endpoint on $\partial M$ and the other in $M$ on which $(y-g) \cdot n=0$. 
The arguments following (2.8) may be applied to show that the segment can be extended to a $C^{\prime \prime}$ curve with both ends on $\partial M$ since $y-g$ never vanishes. Simply replace $Z$ by $Z_{g}$ and $y$ by $y-g$. We must, however, insure that $X$ is regular on the segment at each stage of the extension. Since $M+\partial M$ is compact, the left side of (2.3) takes a positive minimum on $M+\partial M$. Therefore we may suppose that $|g|$ is so small that

$$
e \cdot X_{u} \times X_{v}+e \cdot Z_{g u} \times Z_{g v}>0 \text { on } M+\partial M .
$$

Then $X$ is regular at each point where $Z_{g u} \times Z_{g v}=0$ and hence the extension can be completed to a curve ending on $\partial M$. Call this curve $C_{g}$ and call its endpoints, which are on $\partial M, P_{g}$ and $Q_{g}$.

$d Z_{g}=(y-g) \times d X$ and $(y-g) \cdot n=0$ along $C_{g}$ imply that $d Z_{g}=\lambda n$ for some scalar $\lambda$. Since $(y-g) \cdot d n=0$ along $C_{g}$ and $d X \cdot d n \neq 0$ by (2.7), $\lambda$ cannot be zero at any point of $C_{g}$ for then $y-g$ would be parallel to $d X$ yielding a contradiction. It follows from (2.9) that $e \cdot d Z_{g}=\lambda e \cdot n \neq 0$ on $C_{g}$.

Therefore $d\left(e \cdot Z_{g}\right) \neq 0$ so $e \cdot Z_{g}$ is strictly monotonic on $C_{g} \cdot y$ may vanish but, since it does not vanish in a neighborhood of $P$, this argument (with $g=0$ ) shows that $e \cdot Z$ is monotonic on the curve represented by $X(h(v, 0), v)$ for $0 \leqslant v<a$, for some sufficiently small $a$.

The function $u=h(v, g)$ is continuous for $(v, g)$ in some closed neighborhood of $(0,0)$. Therefore as $g \rightarrow 0, h(v, g) \rightarrow h(v, 0)$ for $v$ in the closed interval $I: 0<v \leqslant$ $a$, if $a$ is sufficiently small. Hence $X(h(v, g), v) \rightarrow X(h(v, 0), v)$ for $v$ in $I$.

Furthermore, since $e \cdot z_{g}$ is continuous, the monotonicity, on $I$, of $e \cdot Z_{g}$ has the same sense for all $g$, if $|g|$ is sufficiently small. We may suppose, without loss of generality, that the monotonicity is increasing. Hence the value of $e \cdot Z_{g}$ at $X(h(a, g), a)$ is positive and bounded from zero, say $>k>0$, as $g \rightarrow 0$. Hence $e \cdot Z_{g}>k>0$ at $Q_{g}$ on $\partial M$.

Let $g \rightarrow 0$ through a sequence $\{g\}$ of values for which $y-g$ never vanishes on $M+\partial M$. Then the corresponding sequence $\left\{Q_{g}\right\}$ has an accumulation point on $\partial M$ at which $e \cdot Z>0$. This contradicts the boundary condition H4. Hence (2.5) holds.

Differentiate the second equation of (2.5) along $\partial M$ to obtain $e \cdot X_{v u}^{\prime}=e \cdot X_{v u}^{\prime \prime}$ and hence by the Gauss equations $\Gamma_{12}^{1} e \cdot X_{u}^{\prime}+\Gamma_{12}^{2} e \cdot X_{v}^{\prime}+M^{\prime} e \cdot n^{\prime}=\Gamma_{12}^{1} e \cdot X_{u}^{\prime \prime}+$ $\Gamma_{12}^{2} e \cdot X_{v}^{\prime \prime}+M^{\prime \prime} e \cdot n^{\prime \prime}$. By (2.5) and the boundary conditions (2.1) we have, since $e \cdot n^{\prime}=e \cdot n^{\prime \prime} \neq 0, M^{\prime}=M^{\prime \prime}$ on $M$. Similarly, differentiating (2.1) along $\partial M$ and using (2.5), we get $L^{\prime}=L^{\prime \prime}$ on $\partial M$. From $L^{\prime} N^{\prime}-M^{\prime 2}=L^{\prime \prime} N^{\prime \prime}-M^{\prime \prime 2}$ it follows that $N^{\prime}=N^{\prime \prime}$ and hence that the boundary strips of $S^{\prime}$ and $S^{\prime \prime}$ are congruent (under rotations and translations).

The result can now be shown to follow, for example, from [2, Theorem I, p. 160], which employs an integral identity but it is instructive to prove it entirely by the present techniques.

If the surfaces are simply connected the theorem follows immediately from the Main Result of [3] which is proved by the method of this paper and which can be stated: 
Let two compact, isometric surfaces be given having positive Gauss curvature. If the surfaces have their normal spherical images in the same compact subset of a hemisphere of the unit sphere and if the isometry is the identity on the boundary then the isometry is the identity.

If the surfaces are not simply connected place the surfaces by a rigid motion (including, possibly, a reflection) preserving the glidebending boundary condition so that one pair of corresponding boundary components coincides. The surface strips of this pair can be assumed to coincide as well so that the second fundamental forms have the same sign, say positive definite, on the boundary components and hence on the whole surfaces.

Let $C_{0}$ be the corresponding boundary component of the parameter manifold $M$. Then $z_{u} \times z_{v}=0$ on $C_{0}$, so by (2.3) the mean surface is regular in a neighborhood $N$ of $C_{0}$.

As in the arguments above following (2.8) and (2.9) we replace $z$ by an equivalent bending field $z_{f}=z-f \times X$, where $f$ is a constant vector close enough in direction to that of $\pm e$ so that $f \cdot n \gtrless 0$ on $M+\partial M$ and so that $y-f$ never vanishes. There exist such vectors with $|f|$ arbitrarily small.

Using those arguments it is easily shown that there exist vectors $f$ so that $z_{f u} \times z_{f v}$ does not vanish on $M . X$ is regular on $N$; therefore by (2.4)

$$
z_{f u} \times z_{f v}=\left[(Y-f) \cdot X_{u} \times X_{v}\right](Y-f) .
$$

$(Y-f) \cdot X_{u} \times X_{v}$ cannot vanish on $N$ if $f$ is chosen as above without contradicting the boundary condition H4. But $Y \cdot X_{u} \times X_{v}=0$ on $C_{0}$ so that the expression in brackets is negative or positive on $\partial M$ and hence on $N$ according as the direction of $f$ or $-f$ is close to that of $e$. Since $|f|$ is arbitrarily small $Y \cdot X_{u} \times X_{v} \equiv$ 0 on $N$. Hence $Y \cdot n$ vanishes on $N$ and hence, by a familiar open-closed argument, on $M$.

Therefore $Y \cdot n_{u} \equiv 0$ and $Y \cdot n_{v} \equiv 0$ on $M$. Since the second fundamental forms of the surfaces are positive definite the Gauss curvature of the mean surface is positive at regular points. Thus $n, n_{u}$ and $n_{v}$ are linearly independent and hence $Y \equiv 0$. Hence $z$ is constant on $M$ and the constant must be zero by definition of $C_{0}$.

3. Application to general caps and caps. The surfaces $S^{\prime}$ and $S^{\prime \prime}$ need not, under the hypotheses of Theorem 1, have a one-to-one orthogonal projection on a plane with normal direction $e$.

In [3] an example is given of a tubular surface with boundary, constructed on a cylindrical helix, which satisfies the conditions of the theorem but is neither convex, i.e., is not a subset of a complete convex surface, nor has a representation in the form $z=f(x, y)$ so that Pogorelov's methods in [5] would not apply.

The helix is given by the position vector

$$
X(t)=a(i \cos t+j \sin t+c t k)
$$

where $i, j, k$ are orthonormal basis vectors and $a$ and $c$ are positive constants. Let $X(v)$ be a representation of the helix in terms of arclength $v$, let $v_{1}, v_{2}, v_{3}$ be the 
Frenet triple of the helix, and let $r$ be a positive constant. Then

$$
X(u, v)=X(v)+r\left(v_{2}(v) \cos u+v_{3}(v) \sin u\right)
$$

is a tubular surface whose cross-sections by the normal planes of the helix are circles of radius $r$. If $\pi+\varepsilon \leqslant u \leqslant \frac{3}{2} \pi-\varepsilon, 0 \leqslant v \leqslant d$, then the conditions of Theorem 1 are satisfied if $\varepsilon$ is a small enough positive number, $r<\kappa^{-1}$, where $\kappa$ is the curvature of the helix, and $d$ is sufficiently large. See [3] for details.

If we further require that $S^{\prime}$ and $S^{\prime \prime}$ have a one-to-one projection on a plane with unit normal $e$ and are simply connected then $S^{\prime}$ and $S^{\prime \prime}$ become what [2, p. 166] defines as general caps (German allgemeine Mützen). That is, a general cap satisfies this requirement and, in addition, $\mathrm{H} 1$ and $\mathrm{H} 2$.

THEOREM 2. Two isometric general caps $X^{\prime}$ and $X^{\prime \prime}$ satisfying the boundary condition $e \cdot X^{\prime}=e \cdot X^{\prime \prime}$ which are oriented so that their unit normals $n^{\prime}$ and $n^{\prime \prime}$ point to their respective concave sides and have $e \cdot n^{\prime}>0$ and $e \cdot n^{\prime \prime}>0$ are congruent under translations and rotations.

A reflection of $X^{\prime}$ or $X^{\prime \prime}$ in a plane parallel to $e$ preserves the boundary condition, reverses the orientation and changes the sign resp. of $e \cdot n^{\prime}$ or $e \cdot n^{\prime \prime}$ so that various equivalent congruence theorems can be stated, both for general caps and the somewhat more general surfaces considered in Theorem 1. For example

TheOREM 3. Two isometric general caps $X^{\prime}$ and $X^{\prime \prime}$ satisfying $e \cdot X^{\prime}=e \cdot X^{\prime \prime}$ on $\partial M$ with mean curvatures $H^{\prime}>0, H^{\prime \prime}<0$ and $e \cdot n^{\prime}>0, e \cdot n^{\prime \prime}<0$ are congruent under rigid body motions and reflections.

The author does not know whether two general caps satisfying $H^{\prime}>0, H^{\prime \prime}<0$, $e \cdot n^{\prime}>0, e \cdot n^{\prime \prime}>0$ on $M+\partial M$ and $e \cdot X^{\prime}=e \cdot X^{\prime \prime}$ on $\partial M$ are congruent. The method of Theorem 1 requires that $H^{\prime}$ and $H^{\prime \prime}$ have the same sign and that $e \cdot n^{\prime}$ and $e \cdot n^{\prime \prime}$ have the same sign. Reflection of $X^{\prime \prime}$ in a plane perpendicular to $e$ would change the sign of $H^{\prime \prime}$ and keep that of $e \cdot n^{\prime \prime}$ but would not preserve the boundary condition in general.

However, if we restrict the boundaries of two isometric general caps to lie in a plane (they are then called simply caps) the conditions of Theorem 1 can be met by rigid motions plus possible reflections (including reflections in the plane of the boundary). Further, in this case it is only necessary to assume that one of the isometric surfaces, say $X^{\prime}$, is a cap. It can be proved that $X^{\prime \prime}$ is a cap provided that it has a plane boundary (see [2, pp. 168-171]) so that we have

THEOREM 4. Let $X^{\prime}$ be a cap with $e \cdot n^{\prime}>0$ and let $X^{\prime \prime}$ be any surface isometric to $X^{\prime}$ which is simply connected, of class $C^{3}$, and has a plane boundary. Then $X^{\prime}$ and $X^{\prime \prime}$ are congruent under rigid body motions and reflections in a plane or, for short:

$A$ cap with spherical image in the interior of a hemisphere allows no finite glidebending.

REMARK. In [2] the weaker condition $e \cdot n^{\prime} \geqslant 0$ is assumed in the preceding theorem. The question of whether a general cap allows a finite glidebending is 
discussed in $[2, \S 80]$ in the context of a possible proof by an integral identity of Grotemeyer but is not proved there.

4. Infinitesimal rigidity. It is simple to use the present method to prove the infinitesimal rigidity of a surface $S^{\prime}$ of the type considered in Theorem 1 , under glidebending since $S^{\prime}$, which plays the role of the mean surface in the proof, is everywhere regular by hypothesis.

THEOREM 5. A surface $S^{\prime}$ which satisfies $\mathrm{H} 1, \mathrm{H} 2$, and $\mathrm{H} 3$ of Theorem 1 is infinitesimally rigid under $a$ bending field $Z$ such that

$\mathrm{H} 4^{\prime}$. $e \cdot Z=0$ on $\partial S^{\prime}$.

REMARK. Infinitesimally rigid means that the only bending fields that $S^{\prime}$ can have are of the trivial form $c \times X+d$, where $c, d$ are constants and $X$ is the position vector of $S^{\prime}$, i.e., the fields are the velocity fields of instantaneous rotations and translations. Two bending fields differing by a trivial bending are equivalent.

A bending field satisfying $\mathrm{H}^{\prime}$ ' is called an infinitesimal glidebending.

As remarked above, a surface of the type of $S^{\prime}$ is more general than a cap, or even general cap, since it need not have a simple projection on any plane or could self-intersect.

SKETCH OF PROOF. Taking $u$ as parameter on $\partial M$, we have $e \cdot y \times X_{u}=0$ so $y$ is a linear combination

$$
y=a e+b X_{u}
$$

on the boundary. If $b$ is identically zero on $S^{\prime}$ then $y_{u}=a_{u} e=\alpha X_{u}-\beta X_{v}$. Since $e \cdot n^{\prime}>0$, the vectors $e, X_{u}$ and $X_{v}$ are linearly independent, so $a_{u}=\alpha=\beta=0$ on $\partial S^{\prime}$. Hence $y$ is equal to a constant $c$ on $\partial S^{\prime} . y-c$ is the rotation field for a bending $Z-c \times X$ equivalent to the original one. Since $y-c$ vanishes on $\partial S^{\prime}$, the new bending is trivial on $S^{\prime}$.

This follows from the result [4, (4.1)], which is proved by the present methods and says, briefly, that if a surface of class $C^{\prime \prime}$ with $K>0$ and spherical image in an open hemisphere has a rotation field $y$ such that $y \cdot n=0$ on the boundary then $y \equiv 0$ on $S^{\prime}$.

Since the new bending is equivalent to the original one, the original is also trivial.

We may therefore assume that $b \neq 0$ at some point $P$ of $\partial S^{\prime}$. Let $a_{P}$ and $b_{P}$ be the values of $a$ and $b$ at $P$. Define $Z^{\prime}=Z-a_{P} e \times X$. $Z^{\prime}$ differs trivially from $Z$, satisfies the boundary condition $e \cdot Z_{u}^{\prime}=0$ and has the rotation field $y^{\prime}=y-a_{P} e$. By $(2.11) y^{\prime} \cdot n=0$ and $y^{\prime} \neq 0$ at $P$. Hence $\left(y^{\prime} \cdot n\right)_{u}$ and $\left(y^{\prime} \cdot n\right)_{v}$ cannot both be zero at $P$, as in the proof of Theorem 1. The rest of the argument is sufficiently similar to the proof of Theorem 1 to be left to the reader. A corollary is

THEOREM 6. A cap with spherical image in an open hemisphere is infinitesimally rigid under glidebending. 


\section{BIBLIOGRAPHY}

1. S. E. Cohn-Vossen, Verbiegbarkeit von Flächen im Grossen, Fortschr. Math. 1 (1936), 33-76.

2. N. W. Efimov, Flächenverbiegung im Grossen mit einem Nachtrag von E. Rembs und $K$. P. Grotemeyer, Akademie-Verlag, Berlin, 1957. MR 19, 59.

3. E. Kann, An elementary proof of a finite rigidity problem by infinitesimal rigidity methods, Proc. Amer. Math. Soc. 60 (1976), 252-258.

4. _ A new method for infinitesimal rigidity of surfaces with $K>0$, J. Differential Geom. 4 (1970), 5-12. MR 41 \#4449.

5. A. V. Pogorelov, Extrinsic geometry of convex surfaces, Nauka, Moscow, 1969; English transl., Transl. Math. Monographs, vol. 35, Amer. Math. Soc., Providence, R.I., 1973. MR 39 \#6222; 49 \#11439.

Department of Mathematics, Queens College (CUNY), Flushing, New York 11367 